\title{
Correlation-Based Fingerprint Matching with Orientation Field Alignment
}

\author{
Almudena Lindoso, Luis Entrena, Judith Liu-Jimenez, and Enrique San Millan \\ University Carlos III of Madrid, Electronic Technology Department, Butarque 15, \\ 28911 Leganes, Madrid, Spain \\ \{alindoso, entrena, jliu, quique\} @ing.uc3m.es
}

\begin{abstract}
Correlation-based techniques are a promising approach to fingerprint matching for the new generation of high resolution and touchless fingerprint sensors, since they can match ridge shapes, breaks, etc. However, a major drawback of these techniques is the high computational effort required. In this paper a coarse alignment step is proposed which reduces the amount of correlations that should be performed. Contrarily to other alignment approaches based on minutiae or core location, the alignment is based on the orientation field estimations. Also the orientation coherence is used to identify the best areas for correlation. The accuracy of the approach is demonstrated by experimental results with an FVC2000 fingerprint database. The approach is also very well suited for hardware acceleration due to the regularity of the used operations.
\end{abstract}

\section{Introduction}

Fingerprints are widely used in recognition of a person's identity because of its proven uniqueness, stability and universality. Characteristic fingerprint features are generally categorized into three levels [1]. Level 1 features, or patterns, are the macro details of the fingerprint such as ridge flow and pattern type (loop, arch, etc.). Level 2 features are the minutiae, such as ridge bifurcations and endings. Level 3 features include all dimensional attributes of the ridge such as ridge width, shape, pores, incipient ridges, breaks, creases, scars, and other permanent details.

Most commercial Automated Fingerprint Identification Systems (AFIS) are based on Level 1 and Level 2 features. This is because the extraction of Level 3 features requires high resolution images, in the order of 1000 pixels per inch (ppi). However, Level 3 features are also claimed to be permanent, immutable and unique according to the forensic experts, and can provide discriminatory information for human identification. With the advent of high resolution fingerprint sensors and "touch-less" sensors that eliminate skin deformation, such as those introduced by TBS, Inc [2], recognition considering Level 3 features becomes feasible. However, many of the existing fingerprint matching techniques cannot take full advantage of these new sensors since they barely consider Level 3 features.

This work focuses on correlation-based fingerprint matching. Correlation uses the gray level information of the fingerprint image since a gray-level fingerprint contains 
much richer, discriminatory information than only the minutiae locations. Correlationbased techniques can take into account Level 3 features as well as other fingerprint features. As a matter of fact, these methods have been used successfully for fingerprint matching with conventional sensors, as demonstrated in the last two fingerprint verification competitions (FVC) [3], [4].

Approaches to correlation-based fingerprint matching have already been proposed [5], [10]. Among the most important aspects of these techniques are the selection of appropriate areas of the fingerprint image for correlation and the computational effort required to consider translation and rotation between the fingerprint images. In order to take into account displacement and rotation, Ouyang et al. propose the use of a local Fourier-Mellin Descriptor (FMD) [6]. However, since the center of relative rotation between two compared fingerprints is unknown, the local FMD has to be extracted for a large number of center locations. Other works correlate ridge feature maps to align and match fingerprint images [7], but do not consider rotation yet.

In this paper we propose techniques that reduce the correlation effort and minimize the effect of deformation by focusing on high-quality and distinctive fingerprint image regions. To this purpose, we introduce a coarse alignment step based on the correlation of the orientation fields of the fingerprints. The alignment dramatically reduces the correlation search space and is further refined at the final correlation step. Due to the lack of high resolution (1000 ppi) fingerprint databases available in the public domain, experimental results have been conducted with a low resolution database (FVC 2000 DB2). Finally, in order to deal with the high computational requirements of correlation techniques, we propose the use of hardware acceleration techniques. As correlation computations are highly regular, they are much more suitable for hardware acceleration than other approaches [8].

The remaining sections of this paper are organized as follows: Section 2 formulates correlation-based fingerprint matching, Section 3 summarizes the fingerprint preprocessing steps, Section 4 describes the matching algorithm including the alignment and correlation region selection approaches, Section 5 analyses the use of hardware acceleration, Section 6 presents the experimental results and finally Section 7 presents the conclusions.

\section{Correlation-Based Fingerprint Matching}

Cross-correlation, or simply correlation, is a measurement of image similarity. In order to compensate variations of brightness, contrast, ridge thickness, etc. that affect the correlation of fingerprint images, the Zero Mean Normalized Cross Correlation (ZNCC) can be used [11]. ZNCC is defined by the expression:

$$
\operatorname{ZNCC}(x, y, \alpha)=\frac{C C(T-\bar{T}, I(x, y, \alpha)-\bar{I}(x, y, \alpha))}{\|T-\bar{T}\| \cdot\|I(x, y, \alpha)-\bar{I}(x, y, \alpha)\|}
$$

where $\mathrm{CC}$ is the cross-correlation, $\mathrm{T}$ is the template image and $\mathrm{I}(\mathrm{x}, \mathrm{y}, \alpha)$ is the input image shifted by $\mathrm{x}$ and $\mathrm{y}$ pixels in vertical and horizontal directions, respectively, and rotated by an angle $\alpha$. 
As an alternative, the cross-correlation required to compute $\mathrm{ZNCC}$ can be obtained by multiplication in the Fourier domain, using the following formula:

$$
C C(T, I)=F^{-1}\left(F^{*}(T) * F(I)\right)
$$

\section{Image Preprocessing}

Correlation-based matching requires good image quality because the matching is performed directly with gray-level fingerprint images. Figure 1 summarizes the preprocessing that has been used in our approach, which is based in [9]. The main steps are: normalization, low frequency noise filtering, orientation field estimation and frequency estimation with their respective coherences, Gabor filtering and finally equalization.

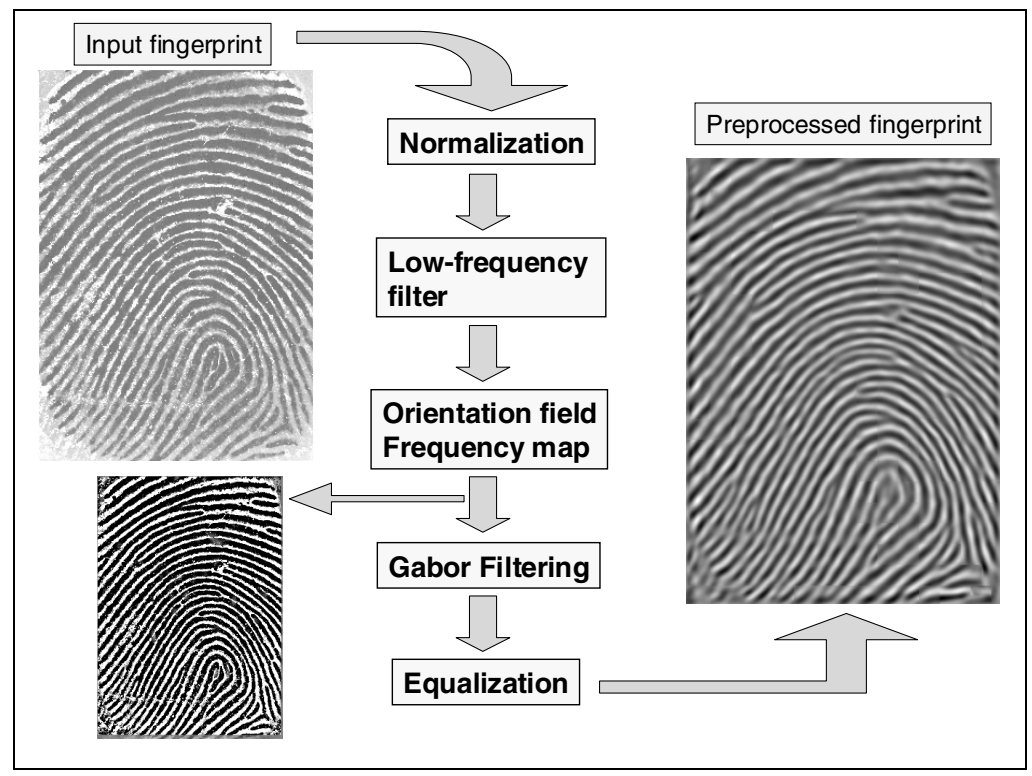

Fig. 1. Preprocessing

\section{Fingerprint Matching}

In the proposed algorithm matching is divided in three steps: image alignment, selection of correlation regions and correlation-based matching.

\subsection{Coarse Alignment}

The purpose of the alignment step is to estimate the translation and rotation between input and template images. This step allows reducing dramatically the amount of 
correlations that should be performed. On the other hand, only a coarse alignment is needed since it will be refined later on in the correlation step.

Most approaches for alignment use features extracted from the images, such as minutiae or core locations [10], [14]. In order to avoid extracting these features, we propose a new approach based on the correlation of the orientation fields computed in the preprocessing step. More precisely, we compute the correlation of the sine and the cosine of the estimated orientation angle weighted by the coherence $(\operatorname{Coh}(\theta))$ as the input image is translated by $(\mathrm{x}, \mathrm{y})$ pixels and rotated by an angle $\alpha$ with respect to the template image:

$$
\begin{aligned}
& C C_{\sin }(x, y, \alpha)=C C\left(\sin \left(2 \theta_{T}\right) * C o h\left(\theta_{T}\right), \sin \left(2 \theta_{I}^{x, y, \alpha}\right) * C o h\left(\theta_{I}^{x, y, \alpha}\right)\right) \\
& (\Delta x, \Delta y, \Delta \alpha)=\max _{x, y, \alpha}\left(C C_{\sin }(x, y, \alpha)+C C_{\cos }(x, y, \alpha)\right) / C C_{C o h}(x, y, \alpha)
\end{aligned}
$$

Note that as the orientation field is rotated, the orientation angle $\theta_{I}^{x, y, \alpha}$ must be corrected by the rotation angle. The correlation maximum determines the best translation $(\Delta \mathrm{x}, \Delta \mathrm{y})$ and rotation $(\Delta \alpha)$. The computational effort required for this correlation operation is acceptable since the orientation map is much smaller than the image. A similar alignment approach could be devised based on the estimation of ridge frequency. However, the results are usually much worse.

\subsection{Selection of Regions}

After alignment, both fingerprints are analyzed in order to determine candidate regions for correlation. Selection of local regions for correlation is required, since using the entire fingerprint will be computationally very expensive and will correlate badly due to fingerprint deformation and noise. On the other hand, the local regions should be highly distinctive. Several approaches for selecting local regions are discussed in [5]. A typical way to choose region candidates consists in computing auto-correlation of the image in order to determine the more distinguishable parts of the image. However, this approach requires a huge computational effort. Regions around core or regions where ridges have high curvature may be selected as candidates, but correlation results may be bad because these are typically very noisy areas. Besides, core does not appear in all fingerprints.

Our approach for the selection of regions is based on image quality and fingerprint overlapping. The quality of the images is an important factor in the search of region candidates for correlation computation. If the region candidate chosen corresponds to a bad quality area in any of the fingerprints, then the verification will fail. To this purpose we use the coherence of the orientation field as a measure of quality [12]. In particular, the multiplication of the coherence of input and template fingerprints is our basic criterion to select region candidates. This computation is only considered for the overlapping areas of both fingerprints, which can be computed thanks to the relative translation and rotation estimated in the alignment step. Notwithstanding, this approach can lead to select regions without enough distinction. To avoid this problem, an average filter is applied to the coherence map, thus allowing the selection of regions with lower coherence as long as they have high coherence neighbours. This correction increases the chances to select distinctive regions located in good quality areas of the image. 
It must be pointed out that if two samples of the same finger are excessively rotated or translated, insufficient overlapping may occur because the images could show non-coincident parts of the same finger. In addition, the overlapping areas may have low quality. In such a case, the matching is attempted with the overlapping portion of the selected region, but the chances the verification fails increase.

\subsection{Matching}

Finally, in the matching step the input region candidates are correlated with the template using ZNCC formula described in section 2. An area, called search area, corresponding to the input region but a little larger is chosen in the template image to perform the correlation. Figure 2 illustrates the region selection process and the main matching steps. Matching is done considering translation and rotation of the input region over the search area. Thanks to the previous alignment step, translation and rotation can be performed at a fine scale within a reduced searching space. In particular, 12 rotations are considered, 6 clockwise and 6 counterclockwise with an angle step of $2^{\circ}$.

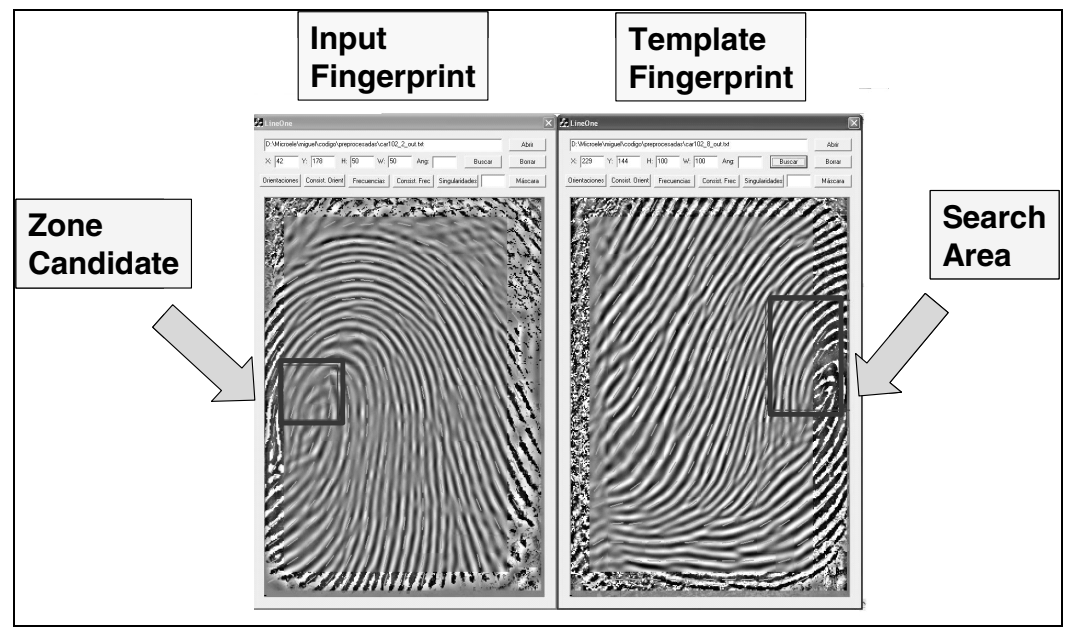

Fig. 2. Region selection and matching steps

\section{Hardware Acceleration}

The proposed fingerprint verification approach relies basically in correlation computations, which are used for the alignment and the matching steps. As correlation computations are highly regular, they are much more suitable for hardware acceleration than other approaches.

Recent results presented in [8] demonstrate that correlation can be accelerated using a FPGA by more than 600 times with respect to a modern PC. This result is obtained by taking advantage of specialized digital signal processing hardware modules, known as DSP slices [16], which are available in modern FPGAs. 
The hardware architecture designed to accelerate correlation computations is summarized in Fig. 3. The architecture follows equation (1) to compute CC as a series of multiply-accumulate (MAC) operations. This approach adapts easily to a variety of correlation sizes, as required for the alingment and the matching steps. The input fingerprint image is stored in the input memory. The template fingerprint image is stored in the input registers of the DSP slices. DSP slices are organized in a matrix, where each slice computes a MAC and passes the result to next slice in the same row. In other words, each row is organized as a systolic array that computes a row correlation. The correlation matrix results from the addition of $n$ consecutive row correlations $\mathrm{R}(\mathrm{i}), \mathrm{i}=0, \ldots, \mathrm{n}-1$. A delay line is inserted after the last slice in every row in order that the result $\mathrm{R}(\mathrm{i})$ reaches the first slice in the next row at the required time to be added up. With this approach, a single data is read from the input memory at every clock cycle, which is supplied to the first slice in each row. At the output of the last row, a correlation result is obtained at every clock cycle for the possible displacements of the input image with respect to the template image.

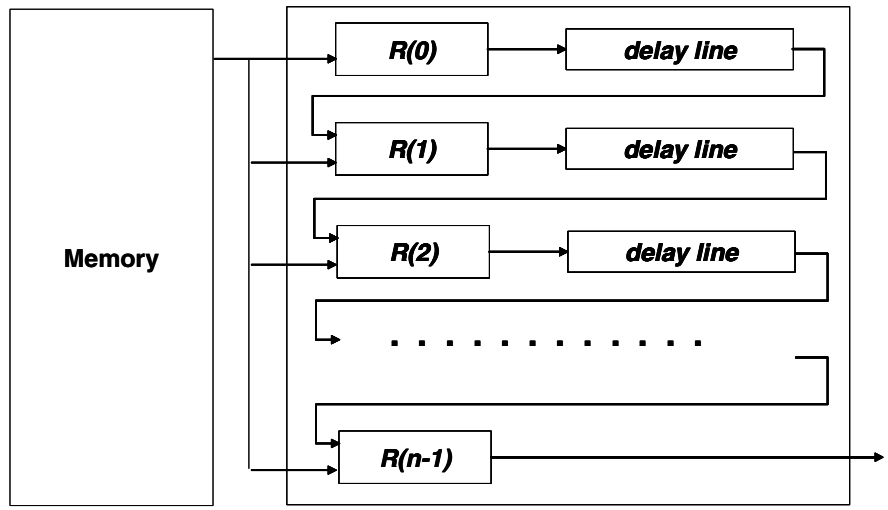

Fig. 3. Hardware architecture for correlation computation

The proposed architecture can be easily scaled to any number of DSP slices and can be implemented with an FPGA or in an ASIC. In practice, the acceleration requirements for real time applications are usually moderate and can be achieved with a low cost FPGA or application-specific hardware in a cost effective manner.

\section{Experimental Results}

The proposed algorithm has been tested with FVC 20002 A [13] data base. This database consists of 100 different fingers, with 8 samples per finger, giving a total of 800 fingerprints. The image size is $256 \times 364$ pixels. For the computation of the orientation field, fingerprint images are divided into blocks of size $64 \times 64$. The resulting orientation field has blocks of size $18 \times 24$. Initially, several number of correlation regions were considered, but after analyzing the results obtained, the number of regions was set to three. After some preliminary experiments, the size of the region was set to 50x50 pixels and the search area to $100 \times 100$ pixels. 
The tests have been conducted considering the FVC premises [15]. To determine the FMR (False Match Rate) curve, the first sample of each finger has been matched against the first sample of all fingers. To determine the FNMR (False Non Match Rate), all the samples of each finger have been matched among themselves. In our case, symmetric matches have been considered in the results because the proposed algorithm is not symmetric.

The ROC (Receiving Operating Curve) obtained for the proposed algorithm is shown in Figure 4. The EER (Equal Error Rate) achieved is $9 \%$.

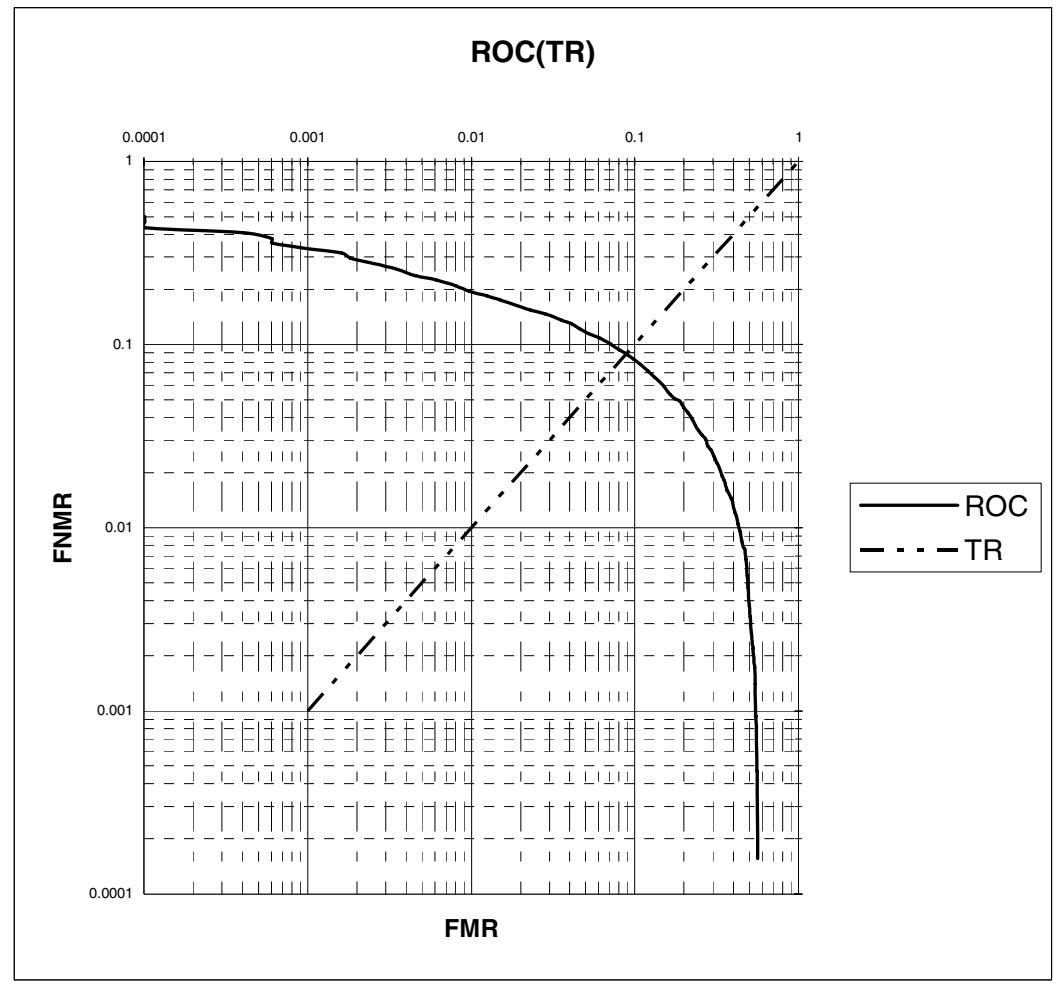

Fig. 4. ROC curve in logarithmic scale of the proposed algorithm tested with FVC 20002 A data Base, FNMR (False Non-Match Rate), FMR (False Match Rate) TR (Correlation Threshold)

The FVC 2000 participants [13] were 11 and their EER range from $0.61 \%$ to $46.15 \%$, for the considered database (DB2). Comparing our results with the participants' results, the EER of the proposed algorithm is below the EER achieved by five of the participants and above the EER achieved by 6 of the participants.

Most of the false matches were due to very bad quality images or insufficient overlapping between the images. These cases can be solved with additional preprocessing steps and particularly, with slightly higher resolution images. With the image size used in the experiments, the correlation regions contain a significant 
portion of the image. This sometimes prevents avoiding low quality areas or using fully overlapped correlation areas of the required size. Also, these cases can be detected at the preprocessing step or later by setting thresholds in the alignment and correlation steps. In a practical application, the verification should be rejected if the image quality is bad or the overlapping is not sufficient, asking the user for a new fingerprint sample. However, this option has not been used in our tests.

The time required to complete the computation for each single verification is 1.15 seconds, divided in 0.25 seconds for preprocessing and 0.9 seconds for matching. This time has been measured with a PC Pentium IV $3 \mathrm{GHz}$ with $2 \mathrm{~GB}$ of RAM for a $\mathrm{C}$ implementation of the algorithm.

The preprocessing time of the proposed algorithm is below the enrollment time reported for all the participants of FVC 2000 [15], considering DB2. Considering both preprocessing and matching times, the total time required for verification is below the time reported for nearly all participants, since only two of them achieve better times.

The implementation of the proposed algorithm can be largely optimized. It must be noted that, given a set of parameter values (block size, region size, search area size, etc.), the computation time is independent of the fingerprints considered, because all computations are completed for every matching. Thus, the computational effort can be significantly reduced by aborting a step as soon as a decision can be made in the alignment and region selection steps.

Correlation computations for the coarse alignment step contribute to the matching time with $109 \mathrm{~ms}$ and correlation computations for matching contribute with $320 \mathrm{~ms}$. Using hardware acceleration for correlation computations, as proposed in Section 5, these times could be substantially reduced. Correlation for coarse alignment can be completed in $1 \mathrm{~ms}$ and correlation for matching in $8 \mathrm{~ms}$. Including data transfer, the whole correlation computations of the proposed algorithm could be computed in less than 14 ms. These results have been obtained with a XC4VSX55 FPGA [16].

\section{Conclusions}

Correlation-based techniques are a promising approach to fingerprint matching for the new generation of high resolution and touch-less fingerprint sensors. However, a large number of correlation computations must be performed in order to consider the possible translation and rotation between the fingerprint images. In this paper, a coarse alignment step based on the correlation of the orientation fields of the fingerprints has been proposed. This alignment dramatically reduces the correlation search space and is further refined at the final correlation step. The orientation field coherence is used to weight the contribution of the orientation estimations to the alignment and to select appropriate regions for correlation.

The experiments presented in this paper demonstrate that this approach produces acceptable results with low resolution sensors. It can be expected that the matching accuracy will improve with high resolution and touchless fingerprint sensors, as these sensors will be able to show Level 3 features and to reduce deformation.

In the past, one of the main drawbacks of correlation-based fingerprint matching approaches was the high computational effort required. With the proposed approach the computational effort has been reduced to an effort comparable to other techniques. Moreover, since this approach relies basically on correlation computations it is very 
suitable for hardware acceleration in order to reduce the verification time. To this purpose, a hardware acceleration architecture has also been proposed that is able to reduce the computational effort required by correlation by two orders of magnitude.

\section{References}

1. Jain, A., Chen, Y., Demitrius, M.: Pores and Ridges: Fingerprint Matching using Level 3 Features. In: Proc. 18th Int'l Conf. on Pattern Recognition (ICPR'06), vol. 4, pp. 477-480 (2006)

2. Parziale, G., Diaz-Santana, E.: The Surround Imager: A Multicamera Touchless Device to Acquire 3D Rolled-Equivalent Fingerprints. In: Zhang, D., Jain, A.K. (eds.) Advances in Biometrics. LNCS, vol. 3832, pp. 244-250. Springer, Heidelberg (2005)

3. Maio, D., Maltoni, D., Cappelli, R., Wayman, J.L., Jain, A.K.: FVC 2002: Second Fingerprint Verification Competition. In: 16th Proc. Int. Conf. on Pattern Recognition, vol. 3, pp. 811-814 (2002)

4. Maio, D., Maltoni, D., Cappelli, R., Wayman, J.L., Jain, A.K.: FVC 2004: Third Fingerprint Verification Competition. In: Proc. Int. Conf. on Biometric Authentication (2004)

5. Bazen, A.M., Verwaaijen, G.T.B., Gerez, S.H., Veelenturf, L.P.J., van der Zwaang, B.J.: A Correlation-Based Fingerprint Verification System. In: Proc. Workshop on Circuits, Systems and Signal Processing (ProRISC), pp. 205-213 (2000)

6. Ouyang, Z., Feng, J., Su, F., Cai, A.: Fingerprint Matching with Rotation-Descriptor Texture Features. In: Proc. 18th Int'l Conf. on Pattern Recognition (ICPR'06), vol. 4, pp. 417-420 (2006)

7. Ross, A., Reisman, J., Jain, A.: Fingerprint Matching using Feature Space Correlation. In: Tistarelli, M., Bigun, J., Jain, A.K. (eds.) ECCV 2002. LNCS, vol. 2359, pp. 48-57. Springer, Heidelberg (2002)

8. Lindoso, A., Entrena, L., Ongil-Lopez, C., Liu, J.: Correlation-based fingerprint matching using FPGAs. In: Proc. Int. conf. on Field Programmable Technology, FPT, pp. 87-94 (2005)

9. Hong, L., Wan, Y., Jain, A.: Fingerprint image enhancement: algorithm and performance evaluation. IEEE Transactions on Pattern Analysis and Machine Intelligence 20(8), 777 789 (1998)

10. Maltoni, D., Maio, D., Jain, A.K., Prabhakar, S.: Handbook of Fingerprint Recognition. Springer, New York (2003)

11. Crouzil, A., Massip-Pailhes, L., Castan, S.: A New Correlation Criterion Based on Gradient Fields Similarity. In: Proc. 13th Int. Conf. on Pattern Recognition, pp. 632-636 (1996)

12. Lim, E., Toh, K.A., Suganthan, P.N., Jiang, X., Yan, W.Y.: Fingerprint quality analysis. In: Proceedings on International Conference on Image Processing, pp. 1241-1244. IEEE, Los Alamitos (2004)

13. Maio, D., Maltoni, D., Cappelli, R., Wayman, J.L., Jain, A.K.: FVC2000: Fingerprint Verification Competition. IEEE Transactions on Pattern Analysis Machine Intelligence 24(3), 402-412 (2002)

14. Park, C.H., Oh, S.K., Kwak, D.M., Kim, B.S., Song, Y.C., Park, K.H.: A new reference point detection algorithm based on orientation patron labelling in fingerprint images. In: Perales, F.J., Campilho, A., Pérez, N., Sanfeliu, A. (eds.) IbPRIA 2003. LNCS, vol. 2652, pp. 697-703. Springer, Heidelberg (2003)

15. Wayman, J., Jain, A., Maltoni, D., Maio, D.: Biometric Systems, Technology, Design and Performance Evaluation. Springer, London (2005)

16. Virtex-4 Family Overview (2004), www.xilinx.com, Xilinx Inc. 